\title{
The combination of angiogenesis and blood vessel invasion as a prognostic indicator in primary breast cancer
}

\author{
T Kato*,', S Kameoka', T Kimura', T Nishikawa ${ }^{2}$ and M Kobayashi ${ }^{3}$ \\ 'Department of Surgery II, School of Medicine, Tokyo Women's Medical University, 8-I Kawadacho, Shinjuku-ku, Tokyo I 62-8666, Japan; ²Department \\ of Surgical Pathology, School of Medicine, Tokyo Women's Medical University, 8-I Kawadacho, Shinjuku-ku, Tokyo I 62-8666, Japan; ${ }^{3}$ Department of \\ Pathology, School of Medicine, Tokyo Women's Medical University, 8-I Kawadacho, Shinjuku-ku, Tokyo I62-8666, Japan
}

\begin{abstract}
This study was undertaken to examine the interaction between the combination of angiogenesis and blood vessel invasion (BVI) and haematogenous metastasis, and to determine the prognostic significance of that combination in predicting 20-year relapse-free survival (RFS) and overall survival (OS) rates in primary breast cancer. Five hundred and nine patients were studied. We investigated I I factors, including average microvessel count (AMC)/BVI, lymph-node status ( $n$ ), clinical tumour size ( $T$ ), histological grade (HG), lymphatic vessel invasion (LVI), p53, proliferating cell nuclear antigen (PCNA), c-erbB-2, mitotic index (MI), apoptotic index, and tumour necrosis (TN). Blood vessel invasion was detected by both factor VIII-related antigen and elastica van Gieson staining. To evaluate the best objective method to quantify microvessel density in angiogenesis, AMC was employed. The rate of AMC-high and BVI-positive tumours was 32.6 and 29.3\%, respectively. That of both AMC-high and BVI-positive tumours was $10.1 \%$. Univariate analysis showed that AMC/BVI, n, T, HG, LVI, p53, PCNA, MI, and TN were significantly predictive of RFS and OS. By multivariate analysis, AMC/BVI was the strongest independent prognostic factor for 20-year RFS (relative risk $(\mathrm{RR})=5.5 ; P<0.000 \mathrm{I}$ ) and for 20-year $O S(R R=4.3 ; P<0.000 I)$. Lymph-node status was still considered a powerful prognostic indicator; however, the combination of $\mathrm{AMC}$ and $\mathrm{BVI}$ provided more reliable prognostic information than lymph-node status for haematogenous dissemination. British Journal of Cancer (2003) 88, 1900 - 1908 doi:I0.I038/sj.bjc.660092I www.bjcancer.com
\end{abstract}

(c) 2003 Cancer Research UK

Keywords: angiogenesis; blood vessel invasion; breast cancer; haematogenous dissemination; long-term survival; microvessel density

There have been many reports about the prognostic factors in breast cancer of which lymph-node status remains a major conventional prognostic factor and has been clinically used as a measure for disseminating the disease, while some investigators have reported that lymph-node metastases have not necessarily been associated with haematogenous dissemination (Fisher et al, 1981; Menard et al, 1994; Braun et al, 2000). Moreover, several additional biologic markers, such as angiogenesis, p53, proliferating cell nuclear antigen (PCNA), c-erbB-2, apoptosis, mitosis, and necrosis have been related to tumour proliferation and development, but their effects on tumour haematogenous metastasis are by no means clear, and there are still controversies regarding the best possible prognostic index for breast cancer patients (Thor et al, 1992; Gilchrist et al, 1993; Zhang et al, 1998; Kato et al, 1999; Beenken et al, 2001; Sirvent et al, 2001).

Some studies have suggested that microvessel density indicated representative angiogenesis of the tumour and was an independent and highly significant prognostic factor for both node-negative and node-positive patients (Weidner et al, 1991; Gasparini et al, 1994; Simpson et al, 1996; Heimann et al, 1996; Kato et al, 2001). However, other authors reported that angiogenesis did not predict

\footnotetext{
*Correspondence: Dr T Kato, Onuma Hospital, 2-10-2, Higashimizumoto, Katsushika-ku, Tokyo I25-0033, Japan;

E-mail: t-kato@bd5.so-net.ne.jp

Received 21 October 2002; revised 30 January 2003; accepted 10 February 2003
}

recurrence in patients with primary breast cancer (Van Hoef et al, 1993; Goulding et al, 1995). The significance of angiogenesis remains controversial because of studies that varied in patient selection, length of time of patient follow-up, antibody used to detect endothelial cells, sample size, method of counting microvessels, and the patients' race.

Recent reports have suggested that peritumour lymphatic and blood vessel invasion (BVI) (vascular invasion) (Lee et al, 1990; Mansi et al, 1991; Fox et al, 1997) or peritumour lymphatic vessel invasion (LVI) (Clemente et al, 1992; Gasparini et al, 1994; Lauria et al, 1996) are significant prognostic factors. However, there are a few recent studies of the prognostic significance of peritumour BVI (Gasparini et al, 1994; Lauria et al, 1996; Kato et al, 2000, 2002). It has been more difficult to identify invasion of small vessels and capillaries and in the larger vessels surrounded by elastic tissue in breast cancer in routine haematoxylin and eosin (H\&E)-stained tissue sections than in gastric or colorectal cancer. A wide range of frequencies has been reported for BVI among patients with breast cancer and the prognostic significance of BVI has not yet been made clear (Weigand et al, 1982; van de Velde et al, 1986; Gasparini et al, 1994; Lauria et al, 1996; Kato et al, 2000, 2002).

Tumours that have acquired the ability of high neovascularisation are likely to involve blood vessels. However, all tumours with high neovascularisation do not have the ability to invade vessels. Gould et al (1983) reported that bronchial carcinoid tumours are highly vascular, but they do not spread beyond the lung. Angiogenesis is necessary for a tumour to grow but not sufficient for it to metastasise. Thus, there are definitely biological 
differences between the ability of tumours to form high neovascularisation and their ability to invade blood vessels. And both the growth of tumours dependent on angiogenesis and the tumours spreading beyond the primary site are dependent on access to the vasculature and are important for haematogenous metastasis.

This study was undertaken to examine the interaction between the combination of angiogenesis and BVI and haematogenous dissemination, and to determine the absolute and relative values of that combination using both Factor VIII-related antigen and elastica van Gieson staining, as we detected by our method published previously (Kato et al, 2001, 2002). Moreover, the clinicopathological significance of p53, PCNA, c-erbB-2, and apoptosis, relatively new prognostic factors, and conventional prognostic factors, especially lymph-node status was evaluated in predicting relapse-free survival (RFS) and overall survival (OS) rates with a long-term follow-up in Japanese patients with breast cancer.

\section{PATIENTS AND METHODS}

\section{Patients}

Data for this study were collected from 509 breast cancer patients selected from those operated on between 1971 and 1990 at Tokyo Women's Medical University Hospital for whom we had sufficient clinical and pathologic material to determine all the biological markers. The distribution of the main clinicopathologic data for the entire 509-patient population is given in Table 1. Two hundred and sixty-four of these patients $(51.9 \%)$ were treated with radical mastectomy, 153 patients $(30.0 \%)$ with modified radical mastectomy, and 92 patients $(18.1 \%)$ with extended radical mastectomy. Although we had no formal protocol for adjuvant therapy, adjuvant chemotherapy was administered to patients with T2, T3 or lymph-node positive tumours. Two hundred and ninety-two of those patients $(57.3 \%)$ received adjuvant chemotherapy including mitomycin C (given intravenously after mastectomy; total doses ranged from 20 to $40 \mathrm{mg}$ for 4-6 weeks) and/or 5-fluorouracil (or its derivatives) were taken orally for 5 years. Postmenopausal patients with positive oestrogen receptors were treated with adjuvant hormone therapy from 1983. Ninety-eight patients (19.3\%) received adjuvant chemotherapy and hormone therapy, and 25 patients $(4.9 \%)$ received adjuvant hormone therapy. No adjuvant treatment was given to 94 patients $(18.5 \%)$. The median follow-up duration of the patients was 9 years (range, 1-20).

\section{Pathological studies}

The original histologic sections of biopsy and mastectomy specimens were reviewed. Paraffin-embedded tissue samples of $4.5 \mu \mathrm{m}$ thick sections stained with $\mathrm{H} \& \mathrm{E}$ were histopathologically assessed. The pathologic specimens were reviewed without any knowledge of the eventual clinical outcome. Conventional clinicopathologic features were observed and recorded, including the clinical tumour size, histological grade, LVI, and tumour necrosis (TN). The clinical tumour size was determined based on the TNM classification, the histological grade was decided based on the Bloom-Richardson grade (Bloom and Richardson, 1957), and LVI was determined based on H\&E staining. Sections of the breast tumour that were stained with $H \& E$ were used to select the maximal area of all the cut surfaces of the tumour that included the invasive component.

Apoptotic cells were assessed in (H\&E) stained sections. The apoptotic cells were defined as cells showing marked condensation of chromatin and cytoplasm, cytoplasmic fragments containing condensed chromatin, and intra- and extracellular chromatin fragments with a diameter of approximately $2 \mu \mathrm{m}$ (Wyllie et al,
Table I Clinicopathologic characteristics of 509 patients

\begin{tabular}{|c|c|}
\hline Characteristics & No. of patients (\%) \\
\hline Patients enrolled & 509 \\
\hline \multicolumn{2}{|l|}{ Age (years) } \\
\hline Median & 49 \\
\hline Range & $15-84$ \\
\hline \multicolumn{2}{|l|}{ Survival follow-up (years) } \\
\hline Median & 9 \\
\hline Range & $1-20$ \\
\hline Recurrence & $103(20.2)$ \\
\hline Deaths & $83(16.3)$ \\
\hline \multicolumn{2}{|l|}{ Menopausal status } \\
\hline Pre & $256(50.3)$ \\
\hline Post & $228(44.8)$ \\
\hline Unknown & $25(4.9)$ \\
\hline \multicolumn{2}{|l|}{ Clinical tumour size $(T)$} \\
\hline TI & $194(38.1)$ \\
\hline T2 & $251(49.3)$ \\
\hline T3 & $64(12.6)$ \\
\hline \multicolumn{2}{|l|}{ Lymph-node status } \\
\hline Negative & $307(60.3)$ \\
\hline Positive & $202(39.7)$ \\
\hline \multicolumn{2}{|l|}{ Average microvessel count } \\
\hline Low & $327(67.4)$ \\
\hline High & $158(32.6)$ \\
\hline \multicolumn{2}{|l|}{ Blood vessel invasion } \\
\hline Negative & $357(70.7)$ \\
\hline Positive & $148(29.3)$ \\
\hline \multicolumn{2}{|l|}{ AMC/BVI } \\
\hline Low/negative & $235(48.7)$ \\
\hline High/negative & $105(21.7)$ \\
\hline Low/positive & $94(19.5)$ \\
\hline High /positive & $49(10.1)$ \\
\hline \multicolumn{2}{|l|}{ Lymphatic vessel invasion } \\
\hline Negative & $387(76.8)$ \\
\hline Positive & $117(23.2)$ \\
\hline \multicolumn{2}{|l|}{ Histological grade } \\
\hline HG I (Well) & $219(43.5)$ \\
\hline HG \| (Moderate) & $|4|(28.0)$ \\
\hline HG III (Poor) & $144(28.5)$ \\
\hline \multicolumn{2}{|l|}{ p53 } \\
\hline Negative & $334(69.7)$ \\
\hline Positive & $145(30.3)$ \\
\hline \multicolumn{2}{|l|}{ c-erbB-2 } \\
\hline Negative & $305(65.3)$ \\
\hline Positive & $162(34.7)$ \\
\hline \multicolumn{2}{|l|}{ PCNA } \\
\hline Negative & $258(53.3)$ \\
\hline Positive & $226(46.7)$ \\
\hline \multicolumn{2}{|l|}{$\mathrm{Ml}$} \\
\hline Negative & $327(65.1)$ \\
\hline Positive & $175(34.9)$ \\
\hline \multicolumn{2}{|l|}{$\mathrm{Al}$} \\
\hline Negative & $397(79.1)$ \\
\hline Positive & $105(20.9)$ \\
\hline \multicolumn{2}{|l|}{ Tumor necrosis } \\
\hline None & $309(6 \mid .6)$ \\
\hline Central & $76(\mid 5.1)$ \\
\hline Comedo & $117(23.3)$ \\
\hline \multicolumn{2}{|l|}{ Histological classification } \\
\hline Infiltrating ductal carcinoma & $466(92.4)$ \\
\hline Infiltrating lobular carcinoma & $20(4.0)$ \\
\hline Others & $18(3.6)$ \\
\hline
\end{tabular}

$\mathrm{PCNA}=$ proliferating cell nuclear antigen; $\mathrm{Ml}=$ mitotic index; $\mathrm{Al}=$ apoptotic index.

1981). Apoptotic cells were counted using a standard light microscope at a 400 magnification ( $\times 40$ objective, $0.152 \mathrm{~mm}^{2}$ per field) in 10 randomised fields. Apoptotic index (AI) was scored as negative ( $<10$ apoptotic cells per 10 fields) and positive $(\geqslant 10$ apoptotic cells per 10 fields). The mitotic index (MI) was determined by a method described in a previous report (Baak 
et al, 1985). The nuclear membrane and the clear zone in the centre had to be absent, and clear, hairy extensions of nuclear material had to be present for them to be counted. Two parallels, and each of the two clearly separate chromosome clumps, were both counted as two mitotic figures. The number of mitotic figures was identified in 10 high-power microscopic fields (objective magnification $\times 40)$. The MI were scored as negative $(<10$ mitotic figures per 10 fields) and positive ( $\geqslant 10$ mitotic figures per 10 fields). The necrosis was divided into two categories. Coagulation necrosis in the centre area of cancer nests was defined as central necrosis, and necrosis in the intraductal component was defined as comedo necrosis (Kato et al, 1997).

\section{Immunohistochemical determinations}

Immunostains for PCNA, p53, c-erbB-2, and factor VIII-related antigen were performed on the paraffin sections using the streptavidin-biotin-immunoperoxidase method as previously described (Kato et al, 1999). Briefly, the primary antibodies employed were an affinity-purified monoclonal anti-human-PCNA antibody (PC10, Novocastra Laboratories, Newcastle, UK) at a $1: 100$ dilution, polyclonal anti-p53 antibody (CM1, Novocastra Laboratories, UK) diluted at a $1: 100$, polyclonal anti-human cerbB-2 protein antibody (Dako, Copenhagen, Denmark) at a 1:100 dilution, and monoclonal antibody (von Willebrand factor $\mathrm{F} 8 / 86$, Dako, Copenhagen, Denmark) applied at 1:200. After immunostaining, the sections were counterstained with haematoxylin. The growth fraction by PCNA staining was evaluated by counting 500 consecutive cells on one slide for each tumour with a $\times 400$ magnification and an index of positive cells to the total number of cells (labeling index (LI)) was made. Labelling index was evaluated and scored as negative ( $<$ mean of LI) and positive ( $\geqslant$ mean of LI). As above, the growth fraction by p53-protein staining was evaluated by counting 500 consecutive cells and LI was made. Labelling index was evaluated and scored as negative $(<$ mean of LI) and positive ( $\geqslant$ mean of LI). For c-erbB-2 protein expression, membrane staining in at least $50 \%$ of the tumour cells was considered positive according to the criteria of Wright et al (1989). Blood vessel invasion was determined using factor VIII-related antigen staining and elastica van Gieson staining as previously described (Kato et al, 2001, 2002). We morphologically classified the BVI into four types according to the patterns of these blood vessels invaded by cancer cells (Figure 1). Type I was a blood vessel directly infiltrated by tumour cells (Figure 1A), type II was a blood vessel filled with tumour cell emboli (Figure 1B-D), type III was a blood vessel with growth of cancer cells between the endothelium and the lamina elastica interna (Figure $1 \mathrm{E}-\mathrm{G}$ ), and type IV was a blood vessel with floating tumour cells (Figure $1 \mathrm{H}$ ). The distinction between lymphatic vessels and blood vessels is difficult and is sometimes arbitrarily determined. While endothelial cells in the lymphatic vessels were occasionally stained by factor VIII-related antigen staining, the pattern of staining in the lymphatic vessels was very faint, discontinuous, and inconsistent in contrast to the intense and continuous staining observed in the vascular endothelium (Figure 1I). On this basis, blood vessels could be differentiated from lymphatic vessels in our study. All doubtful cases were considered to be negative. Blood vessel invasion detected by elastica van Gieson staining was defined by criteria similar to those of Weigand et al (1982). Blood vessels were identified by the following characteristics. Erythrocytes in the lumen, an endothelial cell lining, and the presence of elastic tissue around large vessels and ducts were identified by a zone of acidophilic nonelastic tissue inside the elastic ring surrounding the ducts (Figure 1J). When it was difficult to distinguish BVI from ductal carcinoma in situ (DCIS), the case was considered to be negative. All blood microvessels (capillaries and small venulae) were highlighted by staining endothelial cells with factor VIIIrelated antigen staining. Single brown-stained endothelial cells or clusters of endothelial cells, with or without a lumen, were counted as individual microvessels. To evaluate the best objective method to quantify microvessel density in angiogenesis, the average microvessel count (AMC) per square millimeter was employed (Kato et al, 1999, 2001). One maximal area in one slide of all the cut surfaces exhibiting invasive components in each tumour was scanned at high power $(\times 200)$, and the number of microvessels in the areas along the border between cancer nests and the stroma was recorded. The average number of microvessels in all the fields scanned at high power was calculated giving the mean AMC. The segregation point of the parameter at 54.0 for AMC was determined by the Cox proportional hazards regression model (Cox, 1972).

\section{Statistical analysis}

Statistical analysis of the data was performed with the Survival Tools for Statview-J 4.5 package (Abacus Concepts, Berkeley, CA, USA) on an Apple Power Macintosh 8100/100AV. The association between each parameter was assessed by Pearson's correlation coefficient. We examined the univariate relation between prognostic indicators and 20-year RFS and OS by fitting Kaplan-Meier survival curves (Kaplan and Meier, 1958) to various levels of the prognostic indicators. We then looked for differences among the curves using the log-rank test (Mantel, 1966). The Cox proportional hazards regression model was also used for the multivariate analysis (Cox, 1972).

\section{RESULTS}

\section{Clinical outcome}

Among the 509 patients, there were 103 tumour-related recurrences and 83 tumour-related deaths (Table 1). In addition, there were 23 patients who were lost in the follow-up, 389 patients who are alive, and 14 patients who died of unrelated causes without recurrent tumours, with follow-up examinations of up to 20 years (median, 9 years).

\footnotetext{
Figure I Representative examples of BVI and LVI. (I) Blood vessels directly infiltrated by cancer cells (Type I, A: factor VIII-related antigen staining haematoxylin counter stain, original magnification: $\times 100)$. (2) Blood vessels filled with tumour cell emboli and lymphatic vessel invasion $($ Type II, B: H\&E staining, C: elastica van Gieson staining, D: factor VIII-related antigen staining, haematoxylin counter stain, original magnification: B-D $\times 50$ ). The arrowhead indicates a BVI and arrows indicate LVI with floating tumour cells. The endothelium of lymphatic vessels by factor VIII-related antigen staining was not stained. (3) Blood vessels with growth of cancer cells between endothelium and lamina elastica interna (Type III, E: H\&E staining, F: elastica van Gieson staining, G: factor VIII-related antigen staining, haematoxylin counter stain, original magnification: $E-G \times 50)$. (4) Blood vessels with floating tumour cells (Type IV, $\mathbf{H}$ factor VIII-related antigen staining, haematoxylin counter stain, original magnification: $\times 200)$. (5) The arrowhead indicates a lymphatic vessel with floating tumour cells and arrows indicate blood vessels. The pattern of staining in the lymphatic vessel by factor VIII-related antigen staining was very faint, discontinuous, and inconsistent in contrast to the intense and continuous staining observed in the vascular endothelium (I: factor VIII-related antigen staining, haematoxylin counter stain, original magnification: $\times$ 100). (6) Dilated normal ducts were identified by a zone of acidophilic non-elastic tissue inside the elastic ring surrounding the ducts. The arrowhead indicates the elastic ring and the arrow indicates acidophilic nonelastic tissue. (J: elastica van Gieson staining, original magnification: $\times 50$ ). (7) In non-BVI, the pattern is similar to type III. There is no growth of cancer cells between the endothelium and the lamina elastica interna, while some cancer cells invaded the lamina elastica externa. The arrow indicates cancer cells invading the lamina elastica externa (K: HE staining, L: elastica van Gieson staining, $\mathbf{M}$ : factor VIII-related antigen staining, haematoxylin counter stain, original magnification: $\mathbf{K}-\mathbf{M} \times 50)$.
} 


\section{Pearson's correlation between each parameter}

The rate of AMC-high and BVI-positive tumours was 158 of $485(32.6 \%), 148$ of $505(29.3 \%)$, respectively. That of both AMC-high and BVI-positive tumours was 49 of $483(10.1 \%)$ (Table 1). Table 2 shows the association between each para- meter. When comparing BVI and clinical tumour size, lymphnode status, histological grade, LVI, p53, PCNA, and MI, there were significant correlations among them. On the other hand, AMC weakly correlated with PCNA and c-erbB-2, but AMC/BVI correlated with lymph-node status, LVI, and PCNA (Table 2).
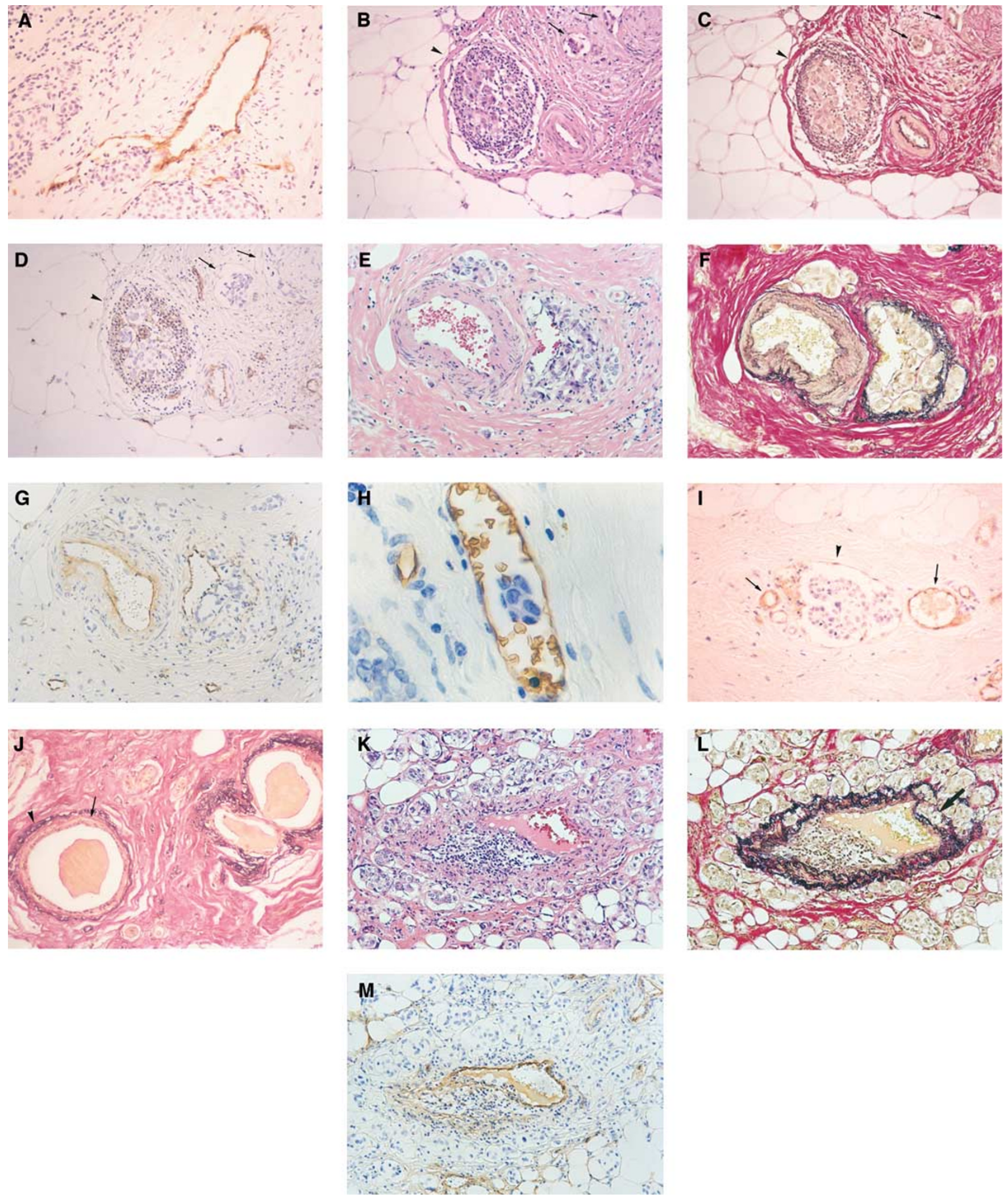
Table 2 Pearson's correlation between each parameter

\begin{tabular}{|c|c|c|c|c|c|c|c|c|c|c|c|c|c|}
\hline Parameter & BVI & AMC & AMC/BVI & $T$ & $n$ & HG & LVI & p53 & PCNA & c-erbB-2 & MI & Al & Necrosis \\
\hline AMC & $\begin{array}{l}0.032 \\
(0.5054)\end{array}$ & I & & & & & & & & & & & \\
\hline$T$ & $\begin{array}{c}0.245 \\
(<0.001)\end{array}$ & $\begin{array}{c}-0.069 \\
(0.1497)\end{array}$ & $\begin{array}{c}0.045 \\
(0.3463)\end{array}$ & I & & & & & & & & & \\
\hline$H G$ & $\begin{array}{c}0.153 \\
(0.0014)\end{array}$ & $\begin{array}{c}-0.148 \\
(0.0019)\end{array}$ & $\begin{array}{c}-0.065 \\
(0.1776)\end{array}$ & $\begin{array}{c}0.319 \\
(<0.001)\end{array}$ & $\begin{array}{c}0.164 \\
(0.0006)\end{array}$ & I & & & & & & & \\
\hline LVI & $\begin{array}{c}0.261 \\
(<0.001)\end{array}$ & $\begin{array}{c}0.083 \\
(0.0828)\end{array}$ & $\begin{array}{c}0.183 \\
(0.0001)\end{array}$ & $\begin{array}{c}0.208 \\
(<0.001)\end{array}$ & $\begin{array}{c}0.422 \\
(<0.00 \mathrm{I})\end{array}$ & $\begin{array}{l}0.048 \\
(0.3182)\end{array}$ & I & & & & & & \\
\hline p53 & $\begin{array}{c}0.145 \\
(<0.001)\end{array}$ & $\begin{array}{c}-0.032 \\
(0.5073)\end{array}$ & $\begin{array}{c}0.031 \\
(0.5|9|)\end{array}$ & $\begin{array}{c}0.107 \\
(0.0257)\end{array}$ & $\begin{array}{c}0.180 \\
(0.0002)\end{array}$ & $\begin{array}{c}0.229 \\
(<0.001)\end{array}$ & $\begin{array}{c}0.035 \\
(0.4703)\end{array}$ & 1 & & & & & \\
\hline PCNA & $\begin{array}{c}0.231 \\
(<0.001)\end{array}$ & $\begin{array}{l}0.099 \\
(0.0386)\end{array}$ & $\begin{array}{c}0.18 \mathrm{I} \\
(0.000 \mathrm{I})\end{array}$ & $\begin{array}{c}0.312 \\
(<0.001)\end{array}$ & $\begin{array}{c}0.447 \\
(<0.001)\end{array}$ & $\begin{array}{c}0.179 \\
(0.0002)\end{array}$ & $\begin{array}{c}0.219 \\
(<0.001)\end{array}$ & $\begin{array}{c}0.179 \\
(0.0002)\end{array}$ & I & & & & \\
\hline $\mathrm{Al}$ & $\begin{array}{l}0.060 \\
(0.2138)\end{array}$ & $\begin{array}{l}-0.014 \\
(0.7719)\end{array}$ & $\begin{array}{c}0.019 \\
(0.6989)\end{array}$ & $\begin{array}{l}0.090 \\
(0.0604)\end{array}$ & $\begin{array}{c}-0.022 \\
(0.6464)\end{array}$ & $\begin{array}{c}0.383 \\
(<0.001)\end{array}$ & $\begin{array}{c}-0.029 \\
(0.5492)\end{array}$ & $\begin{array}{c}0.105 \\
(0.0281)\end{array}$ & $\begin{array}{c}0.089 \\
(0.0625)\end{array}$ & $\begin{array}{c}0.076 \\
(0.1157)\end{array}$ & $\begin{array}{c}0.528 \\
(<0.001)\end{array}$ & 1 & \\
\hline Necrosis & $\begin{array}{l}0.038 \\
(0.4256)\end{array}$ & $\begin{array}{l}-0.131 \\
(0.0063)\end{array}$ & $\begin{array}{c}-0.096 \\
(0.0445)\end{array}$ & $\begin{array}{l}0.140 \\
(0.0035)\end{array}$ & $\begin{array}{c}-0.049 \\
(0.3083)\end{array}$ & $\begin{array}{c}0.370 \\
(<0.001)\end{array}$ & $\begin{array}{l}-0.023 \\
(0.6324)\end{array}$ & $\begin{array}{c}0.167 \\
(0.0005)\end{array}$ & $\begin{array}{c}0.042 \\
(0.3833)\end{array}$ & $\begin{array}{c}0.026 \\
(0.5892)\end{array}$ & $\begin{array}{c}0.478 \\
(<0.001)\end{array}$ & $\begin{array}{r}0.365 \\
(<0.00 I)\end{array}$ & I \\
\hline
\end{tabular}

$\mathrm{BVI}=$ blood vessel invasion; $\mathrm{AMC}=$ average microvessel count; $T=$ clinical tumour size; $n=$ lymph-node status; $\mathrm{HG}=$ histological grade; $\mathrm{LVI}=$ lymphatic vessel invasion; PCNA proliferating cell nuclear antigen; $\mathrm{MI}=$ mitotic index; $\mathrm{Al}=$ apoptotic index; $P$-values are given in parentheses.

A

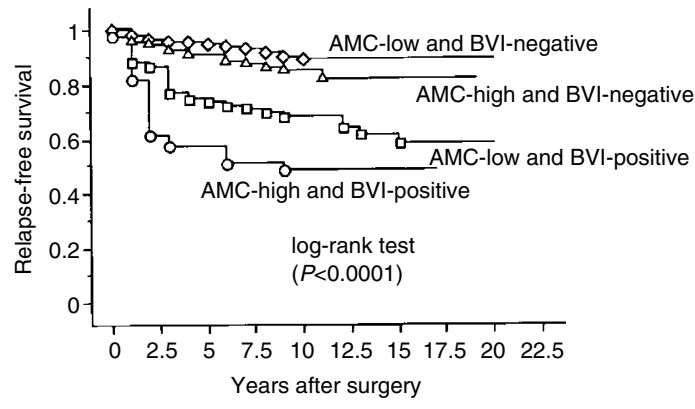

No. at risk

$\begin{array}{lrrrr}\text { AMC-low and BVI-negative } & 235 & 211 & 124 & 38 \\ \text { AMC-high and BVI-negative } & 105 & 87 & 46 & 6 \\ \text { AMC-low and BVI-positive } & 94 & 67 & 38 & 18\end{array}$

AMC-high and BVI-positive $\begin{array}{llll}49 & 27 & 13 & 3\end{array}$

\section{B}

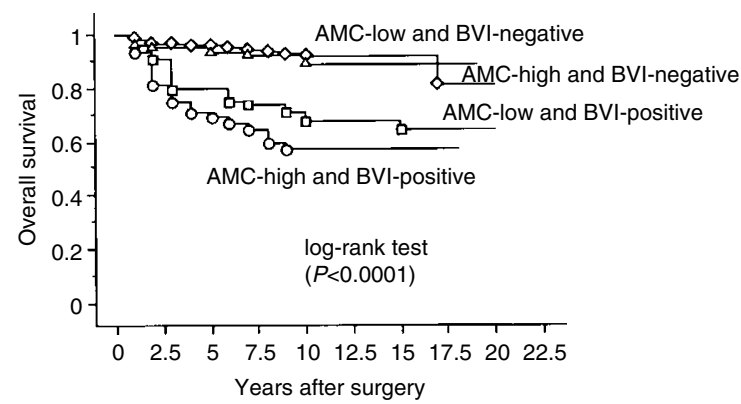

No. at risk

AMC-low and BVI-negative $\quad 235 \quad 213 \quad 126 \quad 39$

AMC-high and BVI-negative $\begin{array}{llll}105 & 91 & 49\end{array}$

$\begin{array}{lllll}\text { AMC-low and BVI-positive } & 94 & 72 & 41 & 20\end{array}$

AMC-high and BVI-positive $\quad 49 \quad 33 \quad 16 \quad 5$

Figure 2 Kaplan-Meier survival curves for all patients. (A) RFS stratified by $\mathrm{AMC}$ and $\mathrm{BVI}$. (B) OS related to $\mathrm{AMC}$ and $\mathrm{BVI}$.

\section{Univariate analysis}

The prognostic factors found to be significantly associated with 20-year RFS were AMC/BVI $(P<0.0001$, Figure $2 \mathrm{~A})$, clinical tumour size, lymph-node status, histological grade, LVI, p53, PCNA, MI, and TN (Table 3). Moreover, AMC/BVI $(P<0.0001$, Figure $2 \mathrm{~B})$, clinical tumour size, lymph-node status, histological grade, LVI, p53, PCNA, c-erbB-2, MI, and TN were associated with 20 -year OS. However, AI was not associated with either 20 -year RFS or OS (Table 3 ).

\section{Multivariate analysis}

Table 4 shows that AMC/BVI was the strongest independent prognostic factor for 20-year RFS (relative risk $(\mathrm{RR})=5.5$; $P<0.0001)$ and for 20 -year OS $(\mathrm{RR}=4.3 ; P<0.0001)$ by multivariate analysis.

\section{Stratification by lymph-node status}

When stratified by lymph-node status, a significant impact of AMC/BVI on 20-year RFS or OS was seen in patients with nodenegative and -positive carcinomas by univariate analysis $(P<0.0001$ or $P<0.0001$, and $P<0.0001$ or $P<0.0001$, respectively). We fitted a model with four factors: AMC/BVI, clinical tumour size, histological grade, and p53, in patients with nodenegative carcinoma, and compared them by multivariate analysis. AMC/BVI was a significant independent factor for 20-year RFS or OS $(\mathrm{RR}=19.1 ; 95 \%$ confident interval $(95 \% \mathrm{CI})=4.6-79.5$; $P<0.0001$, or $\mathrm{RR}=20.3 ; 95 \% \mathrm{CI}=3.3-127.1 ; P=0.0013$, respectively). Moreover, AMC/BVI was a significant independent factor for 20-year RFS or OS in patients with node-positive carcinoma on multivariate analysis $(\mathrm{RR}=3.4 ; 95 \% \mathrm{CI}=1.7-6.7 ; P=0.0005$, or $\mathrm{RR}=2.9 ; 95 \% \mathrm{CI}=1.4-6.2 ; P=0.0049$, respectively). Patients with node-positive carcinoma who had AMC-high and BVI-positive tumours had a higher risk of cancer-related death than the patients with node-negative carcinoma who had AMC-low and BVI- 
Table 3 Univariate analysis of the value of prognostic factors for RFS and OS

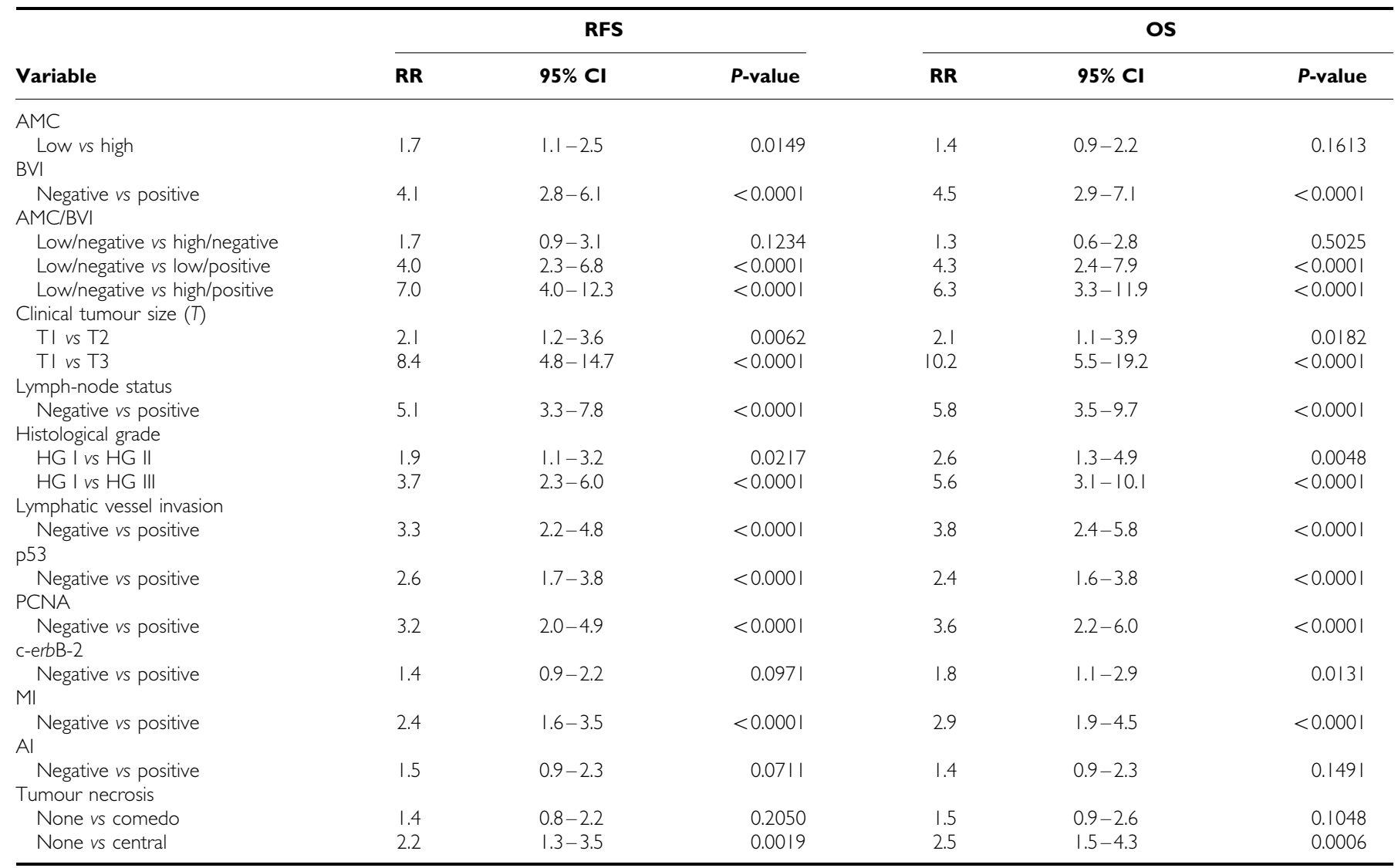

$\mathrm{RFS}=$ relapse-free survival; $\mathrm{OS}=$ overall survival; $\mathrm{RR}=$ relative risk; $\mathrm{AMC}=$ average microvessel count; $\mathrm{BVI}=$ blood vessel invasion; $P C N A=$ proliferating cell nuclear antigen; $\mathrm{MI}=$ mitotic index; $\mathrm{Al}=$ apoptotic index; Hazards ratio from Cox regression analysis.

negative tumours on RFS or OS $(\mathrm{RR}=54.9 ; 95 \% \mathrm{CI}=16.1-186.4$; $P<0.0001, \mathrm{RR}=61.0 ; 95 \% \mathrm{CI}=14.0-266.6 ; P<0.0001$, respectively, Figures $2 \mathrm{~A}$ and $\mathrm{B})$. There was no significant difference in 20 year RFS or OS between patients with node-negative carcinoma who had AMC-high and BVI-positive tumours, and patients with node-positive carcinoma who had AMC-low and BVI-negative tumours $(P=0.9522$ and $P=0.6867$, respectively, Figures $3 \mathrm{~A}$ and $\mathrm{B})$.

\section{Characteristics of the treatment}

We examined the effect of treatment in all patients for RFS and OS. The modality of operation was not associated with RFS or OS. However, adjuvant chemotherapy was significantly associated with OS but not with RFS $(\mathrm{RR}=1.6 ; 95 \% \mathrm{CI}=1.0-2.5 ; P=0.0428$, or $\mathrm{RR}=1.2 ; 95 \% \quad \mathrm{CI}=0.8-1.8 ; \quad P=0.4927$, respectively). Using interaction terms in the Cox proportional hazards model, we examined variations in the importance of AMC/BVI across treatment subgroups. In the subgroup of 94 patients without adjuvant therapy, the relative risk of both RFS and OS also associated with AMC/BVI was significant $(\mathrm{RR}=4.7 ; 95 \% \mathrm{CI}=1.5-$ $14.9 ; P=0.0085$, and $\mathrm{RR}=4.3 ; 95 \% \mathrm{CI}=1.4-13.8 ; P=0.0126$, respectively). On the other hand, in the subgroup of 283 patients with chemotherapy, the relative risk of both RFS and OS associated with AMC/BVI remained significantly elevated $(\mathrm{RR}=6.8 ; 95 \%$ $\mathrm{CI}=3.0-15.4 ; \quad P<0.0001, \quad$ and $\quad \mathrm{RR}=6.7 ; \quad 95 \% \quad \mathrm{CI}=2.6-16.8$; $P<0.0001$, respectively).

\section{DISCUSSION}

Results of the present study confirm AMC/BVI as the strongest independent prognostic factor for 20-year RFS and OS in Japanese patients with breast cancer. AMC/BVI was also a significant independent factor for 20-year RFS or OS in patients with both node-negative and positive carcinomas; especially, the effect of the presence of $\mathrm{AMC} / \mathrm{BVI}$ was clearer in patients with node-negative carcinomas than in those with node-positive carcinomas.

Some clinical studies suggested that to use an antibody to CD31 may be superior to using factor VIII-related antigen (Horak et al, 1992; Fox et al, 1994), however, the other study reported that this greater sensitivity of anti-CD31 of vascular endothelium did not yield more discriminating results for predicting survival outcome than results produced with factor VIII-related antigen (Gasparini et al, 1994). At that point, it may lack some acuracy; however, many stromal vessels can be stained very well and be taken in to account by factor VIII-related antigen staining as we published previously (Kato et al, 1999). When the authors compared AMC with the highest microvessel density in one or three fields (the hot spots) in the previous study, AMC was a more reliable factor than that of the hot spots (Kato et al, 1999). Therefore, we used AMC in this study. The results show that AMC was an independent prognostic factor, but its prognostic impact was not as strong as lymph-node status and clinical tumour size.

The rate of BVI fell within the range of $4.2-52.0 \%$ as observed in other studies (Teel and Sommers, 1964; Friedell et al, 1965; Sampat 
A

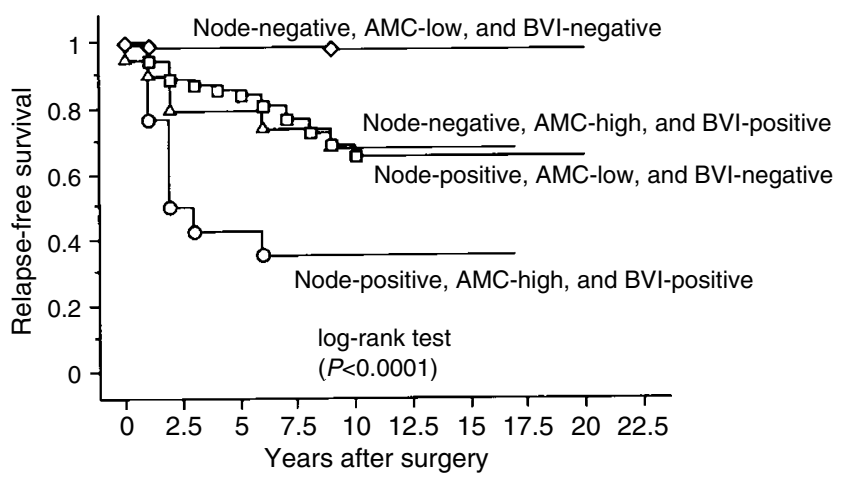

No. of patients at risk

Node-negative, AMC-low, and 163 $158 \quad 100$ BVI-negative

Node-negative, AMC-high, and BVI-positive

Node-positive, AMC-low, and BVI-negative

Node-positive, AMC-high, and BVI-positive

B

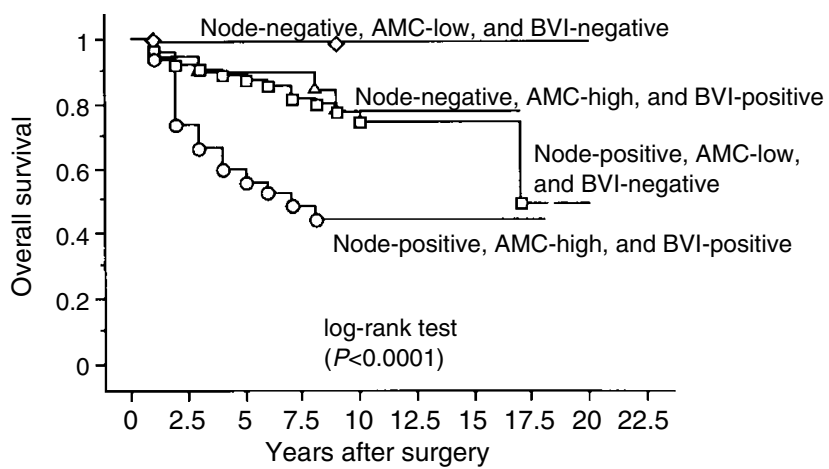

No. of patients at risk

Node-negative, AMC-low, and BVI-negative

Node-negative, AMC-high, and BVI-positive

Node-positive, AMC-low, and

BVI-negative

Node-positive, AMC-high, and BVI-positive

$\begin{array}{rrrr}19 & 15 & 7 & 1 \\ 72 & 33 & 23 & 5 \\ 30 & 12 & 6 & 2\end{array}$

Figure 3 Kaplan-Meier survival curves for AMC-low and BVI-negative and AMC-high and BVI-positive patients. (A) RFS stratified by node, AMC, and $\mathrm{BVI}$. (B) OS related to by node, AMC, and BVI.

et al, 1977; Cooperative Breast Cancer Group, 1978; Martin et al, 1987; Fisher et al, 1993; Kister et al, 1996; Lauria et al, 1996; Kato et al, 2002). In this study, BVI in the breast cancer patients we observed was seen in 148 cases $(29.3 \%)$. On the other hand, the rate of BVI examined by Lauria et al (1996) was $4.2 \%$. They detected BVI using H\&E staining alone, and the prevalence of BVI was particularly low in their study. In the previous study, when we evaluated BVI by H\&E staining alone, the rate of BVI presented in $6.5 \%$, which was similar to the study by Lauria et al (Lauria et al, 1996; Kato et al, 2002). By H\&E staining alone, it was difficult to detect blood vessels filled with tumour cell emboli, to distinguish BVI from pseudoemboli resulting from shrinkage where cancer cells peeled off from the ducts because of poor fixing, to distinguish between small blood vessel invasion and LVI, to find the blood vessels with cancer cells growing between the endothelium and the lamina elastica interna, and to distinguish BVI from DCIS covered with elastic fibre (Figure 1, Kato et al, 2002). Therefore, there was a possibility of overlooking some kinds of blood vessel invasion using H\&E staining alone. When Weigand et al (1982) and others (Sampat et al, 1977) examined the prognostic value of BVI, BVI correlated significantly with a high rate of recurrence. The results of our study suggest that BVI is an adverse prognostic factor among patients with breast cancer as well as lymph-node status. However, when Lauria et al (1996) examined the prognostic value of BVI, it was associated with shorter survival by univariate analysis but not significantly an independent factor by multivariate analysis. These discrepancies may be because of some technical reasons, especially the staining methods of BVI, number of cases studied, the different follow-up period and different races.

Average microvessel count and BVI retained an independent significance in association with RFS or OS by multivariate analysis (Table 4). Independent of lymph-node status, if there were AMClow and BVI-negative tumours, we observed similar, excellent, long-term survival. Moreover, we used both factors to define a high-risk subset of all the tumours in cases that experienced a $48.1 \%$ recurrence or a $57.4 \%$ survival probability within 17 years (Figure 1A and B). There are likely to be worse prognoses when carcinomas acquire the ability to form AMC-high and BVI-positive tumours, which then become more important indications of outcome than lymph-node status and clinical tumour size. Our study suggests that even when tumours acquire a high degree of neovascularisation, it is impossible to form distant metastases without BVI and that AMC/BVI might be the most important and strongest predictive factor for tumour haemtogenous metastasis.

In recent studies, it has been suggested that examination for bone marrow micrometastasis using immunocytochemical methods in breast cancer patients would be useful as a prognostic marker (Braun et al, 2000). Moreover, other investigators reported that an assessment of tumour angiogenesis and vascular invasion give a reliable indication of the likelihood of the presence of bone marrow micrometastasis in patients with breast cancer and both processes contribute to metastases (Mansi et al, 1991; Fox et al, 1997). However, as they did not distinguish BVI from LVI, the meaning of the pathway of haematogenous metastastasis remains unclear. Although some investigators have indicated it impractical to distinguish between the blood and lymphatic vessel systems as independent routes of tumour dissemination because they are so interrelated (Fisher and Fisher, 1966), the present authors examined the difference between BVI and LVI in order to distinguish the different pathways to distant metastases. As vascular invasion includes the routes of both lymphatic and haematogenous dissemination, BVI alone may show haematogenous dissemination more accurately than vascular invasion. Haematogenous metastasis can be considered a complex process of cascading interregulated sequential steps. Average microvessel count and BVI include many steps that are necessary for haematogenous dissemination, that is, they are the results of tumour invasion to extracelluar matrices and basement membranes, entering the circulatory system, and activating angiogenesis at both the primary and distant sites. Our results suggest that tumours can spread directly via the blood-vascular system from their initial growth sites, and that biologically the significance of the combination of AMC and BVI is clearly more important than that of either BVI or AMC alone.

There are some reports that indicate adequate locoregional treatment for early breast cancer may prevent secondary dissemination (Hellman and Harris, 1987; Arriagada et al, 1995). However, Fisher et al (1981) maintained that breast cancer is a systemic disease and positive lymph nodes are not important instigators of distant disease. Several investigators have found no correlation between nodal metastases and bone-marrow 
Multivariate analysis showing independent factors of RFS and OS

\begin{tabular}{|c|c|c|c|c|c|c|c|c|c|c|c|c|}
\hline \multirow[b]{2}{*}{ Model variable } & \multicolumn{3}{|c|}{$\begin{array}{c}\text { I } \\
\text { RFS }\end{array}$} & \multicolumn{3}{|c|}{$\begin{array}{c}2 \\
\text { RFS }\end{array}$} & \multicolumn{3}{|c|}{$\begin{array}{l}3 \\
\text { os }\end{array}$} & \multicolumn{3}{|c|}{$\begin{array}{c}4 \\
\text { OS }\end{array}$} \\
\hline & $\mathbf{R R}$ & $95 \% \mathrm{Cl}$ & $P$-value & $\mathbf{R R}$ & $95 \% \mathrm{Cl}$ & $P$-value & $\mathbf{R R}$ & $95 \% \mathrm{Cl}$ & $P$-value & $\mathbf{R R}$ & $95 \% \mathrm{Cl}$ & $P$-value \\
\hline Low vs high & 2.3 & $1.5-3.5$ & 0.0002 & & & & 1.8 & $1.1-3.0$ & 0.0175 & & & \\
\hline BVI & & & & & & & & & & & & \\
\hline $\begin{array}{l}\text { Negative vs positive } \\
\text { AMC/BVI }\end{array}$ & 2.3 & $1.5-3.6$ & 0.0001 & & & & 2.3 & $1.4-3.8$ & 0.0011 & & & \\
\hline Low/negative vs high/positive & & & & 5.5 & $3.0-10.1$ & $<0.0001$ & & & & 4.3 & $2.2-8.5$ & $<0.0001$ \\
\hline \multicolumn{13}{|l|}{ Clinical tumour size $(T)$} \\
\hline TI vs $\mathrm{T} 2$ & 1.7 & $0.9-3.0$ & 0.0955 & 1.6 & $0.9-2.9$ & 0.1153 & 1.5 & $0.7-3.0$ & 0.3025 & 1.4 & $0.7-2.9$ & 0.3277 \\
\hline TI vs T3 & 3.7 & $2.0-7.2$ & $<0.0001$ & 3.6 & $1.9-7.0$ & 0.0001 & 3.8 & $1.8-7.9$ & 0.0005 & 3.7 & $1.8-7.8$ & 0.0006 \\
\hline \multicolumn{13}{|l|}{ Lymph-node status } \\
\hline Negative vs positive & 3.0 & $|.8-5|$. & $<0.0001$ & 3.0 & $1.8-5.0$ & $<0.0001$ & 3.8 & $2.1-7.1$ & $<0.0001$ & 3.7 & $2.0-6.9$ & $<0.0001$ \\
\hline
\end{tabular}

$\mathrm{RR}=$ relative risk; $\mathrm{RFS}=$ relapse-free survival; $\mathrm{OS}=$ overall survival; $95 \% \mathrm{Cl}=95 \%$ confidence interval; $\mathrm{AMC}=$ average microvessel count; $\mathrm{BVI}=$ blood vessel invasion; The multivariate analysis employing Cox proportional hazard regression of prognostic factors of RFS and OS is shown. In the model regarding RFS five or six prognostic indicators are compared. In the model regarding OS five or six prognostic indicators are compared.

metastases (Harbeck et al, 1994; Menard et al, 1994). Moreover, Menard et al (1994) reported that pathological nodal status was not a more reliable prognostic factor than the primary tumour score, including tumour size, grading, laminin receptor, and c-erbB-2. Our results suggest that tumours can spread directly through the blood-vascular system from their initial growth sites, and that lymph-node status was still considered a powerful prognostic indicator; however, biologically the combination of AMC and BVI

\section{REFERENCES}

Arriagada R, Rutqvist LE, Mattsson A et al (1995) Adequate locoregional treatment for early breast cancer may prevent secondary dissemination. J Clin Oncol 13: 2869-2878

Baak JPA, van Dop H, Kurver HJ, Hermans JO (1985) The value of morphometry to classic prognosticators in breast cancer. Cancer 56: $374-382$

Beenken SW, Grizzle WE, Crowe DR et al (2001) Molecular biomarkers for breast cancer prognosis: coexpression of c-erbB-2 and p53. Ann Surg 233: $630-638$

Bloom HIJ, Richardson WW (1957) Histologic grading and prognosis. $\mathrm{Br} \mathrm{J}$ Cancer 11: 359-377

Braun S, Pantel K, Muller P, Janni W, Hepp F, Kentenich CRM, Gastroph S, Wischnik A, Dimpfl T, Kindermann G, Riethmuller G, Schlimok G (2000) Cytokeratin-positive cells in the bone marrow and survival of patients with stage I, II, or III breast cancer. $N$ Engl J Med 342: 525-533

Clemente CG, Boracchi P, Andreola S, Del Vecchio M, Veronesi P, Rilke FO (1992) Peritumoural lymphatic invasion in patients with node-negative mammary duct carcinoma. Cancer 69: 1396-1403

Cooperative Breast Cancer Group (1978) Identification of breast cancer patients with high risk of early reccurence after radical mastectomy. II. Clinical and pathological correlations. Cancer 42: 2809-2826

Cox DR (1972) Regression models and life tables. J Roy Stat Soc B 34: 187-202

Fisher ER, Costantino J, Fisher B, Redmond C, Collaborating national surgical adjuvant breast bowel project investigators (1993) Pathologic findings from the national surgical adjuvant breast project (protocol 4) Discriminants for 15-year survival. Cancer 71: $2141-2150$

Fisher B, Fisher ER (1966) The inter-relationship of hematogenous and lymphatic tumour cell dissemination. Surg Gynecol Obstet 122: 791 - 798 is more significant than lymph-node status for tumour haematogenous dissemination.

\section{ACKNOWLEDGEMENTS}

We thank Mr Minoru Sakurada and his colleagues for their work in the immunohistochemical staining and Mr Robert E Brandt for his help in editing the manuscript.
Fisher B, Wolmark N, Redmond C, Deutsch M, Fisher ER (1981) Findings from NSABP protocol No. B-04: comparison of radical mastectomy with alternative treatments. II. The clinical and biologic significance of medial-central breast cancers. Cancer 48: 1863-1872

Fox SB, Leek RD, Bliss J, Mansi JL, Gusterson B, Gatter KC, Harris AL (1997) Association of tumour angiogenesis with bone marrow micrometastases in breast cancer patients. J Natl Cancer Inst 89: $1044-1049$

Fox SB, Leek RD, Smith K, Hollyer J, Greenall M, Harris AL (1994) Tumor angiogenesis in node-negative breast carcinomas-relationship with epidermal growth factor receptor, estrogen receptor, and survival. Breast Cancer Res Treat 29: 109-116

Friedell GH, Betts A, Sommers SC (1965) The prognostic value of blood vessel invasion and lymphocytic infiltrates in breast carcinoma. Cancer 18: $164-166$

Gasparini G, Weidner N, Bevilacqua P, Maluta S, Palma PD, Caffo O, Barbareschi M, Boracchi P, Marubini E, Pozza F (1994) Tumour microvessel density, p53 expression, tumour size, and peritumoural lymphatic vessel invasion are relevant prognostic markers in nodenegative breast carcinoma. J Clin Oncol 12: 454-466

Gilchrist KW, Gray R, Fowble B, Tormey DC, Taylor IV (1993) Tumour necrosis is a prognostic predictor for early recurrence and death in lymph-node positive breast cancer: a 10-year follow-up study of 729 Eastern Cooperative Oncology Group patients. J Clin Oncol 11: $1925-1935$

Gould VE, Linnoila RL, Memoli VA, Warren WH (1983) Neuroendocrine components of the bronchopulmonary tract: hyperplasis, dysplasias, and neoplasms. Lab Invest 49: 519-537 
Goulding H, Rashid NFNA, Robertson JF, Bell JA, Elston CW, Blamey RW, Ellis IO (1995) Assessment of angiogenesis in breast carcinoma: an important factor in prognosis? Hum Pathol 26: 1196-1200

Harbeck N, Untch M, Pachet l, Eiermann W (1994) Tumour cel detection in the bone marrow of breast cancer patients at primary therapy: results of 3-year median follow-up. Br J Cancer 69: 566-571

Heimann R, Ferguson D, Powers C, Recant WM, Weichselbaum RR, Hellman S (1996) Angiogenesis as a predictor of long-term survival for patients with node-negative breast cancer. J Natl Cancer Inst 88: $1764-1769$

Hellman S, Harris J (1987) The appropriate breast carcinoma paradigm. Cancer Res 47: 339-342

Horak ER, Leek R, Klenk N, Lejeune S, Smith K, Stuart N, Greenall M, Stepniewska K, Harris AL (1992) Angiogenesis, assessed by platelet/ endothelial cell adhesion molecule antibodies, as indicator of node metastases and survival in breast cancer. Lancet 340: 1120-1124

Kaplan EL, Meier P (1958) Non-parametric estimation from incomplete observations. J Am. Stat Assoc 53: 457-481

Kato T, Kameoka S, Kimura T, Nishikawa T, Kobayashi M (2002) Blood vessel invasion as a predictor of long-term survival for Japanese patients with breast cancer. Breast Cancer Res Treat 73: 1-12

Kato T, Kameoka S, Kimura T, Soga N, Abe Y, Nishikawa T, Kobayashi M (2001) Angiogenesis as a predictor of long-term survival for 377 Japanese patients with breast cancer. Breast Cancer Res Treat 70: 65-74

Kato T, Kimura T, Miyakawa R, Fujii A, Yamamoto K, Kameoka S, Nishikawa T, Kasajima T (2000) Clinicopathological study associated with long-term survival in Japanese patients with node-negative breast cancer. Br J Cancer 82: 404-411

Kato T, Kimura T, Miyakawa R, Nobue I, Fujii A, Yamamoto K, Kameoka S, Toshio N, Kasajima T (1999) The methodology of quantitation of microvessel density and prognostic value of neovascularization associated with long-term survival in Japanese patients with breast cancer. Breast Cancer Res Treat 53: 19-31

Kato T, Kimura T, Miyakawa R, Tanaka S, Fujii A, Yamamoto K, Kameoka S, Hamano K, Kawakami M, Aiba M (1997) Clinicopathologic study of angiogenesis in Japanese patients with breast cancer. World J Surg 21: $49-56$

Kister SJ, Sommers SC, Haagensen CD, Cooley E (1996) Reevaluation of blood vessel invasion as a prognostic factor in carcinoma of the breast. Cancer 19: $1213-1216$

Lauria R, Perrone F, Carlomagna C, De Laurentis M, Morabit A, Gallo C, Varriale E, Pettinato G, Panico L, Petrella G, Bianco AR, De Placido S (1996) The prognostic value of lymphatic and blood vessel invasion in operable breast cancer. Cancer 76: $1772-1778$

Lee AKC, Delellis RA, Silverman ML, Heatley GJ, Wolfe HJ (1990) Prognostic significance of peritumoural lymphatic and blood vessel Invasion in node-negative carcinoma of the breast. J Clin Oncol 18: $1457-1465$

Mansi JL, Easton D, Berger U, Gazet J-C, Ford HT, Dearnaley D, Coombes RC (1991) Bone marrow micrometastasis in primary breast cancer: prognostic significance after 6 years' follow-up. Eur J Cancer 27: $1552-1555$

Mantel N (1966) Evaluation of survival data and two new rank order statistics arising in its consideration. Cancer Chemother Rep 50: $163-170$

Martin SA, Perez-Reyes N, Mendelsonhn G (1987) Angiogenesis in breast carcinoma: an immunohistochemical study of factor VIII-related antigen. Cancer 59: $1918-1922$

Menard S, Bufalino R, Rilke F, Cascinelli N, Veronesi U, Colnaghi MI (1994) Prognosis based on primary breast carcinoma instead of pathological nodal status. Br J Cancer 70: 709-712

Sampat MB, Sirsat MV, Gangadharan P (1977) Prognostic significance of blood vessel invasion in carcinoma of the breast in women. J Surg Oncol 9: $623-632$

Simpson JF, Ahn C, Battifora H, Esteban JM (1996) Endothelial area as a prognostic indicator for invasive breast carcinoma. Cancer 77: 2077-2085

Sirvent JJ, Fortuno-Mar A, Olona M, Orti A (2001) Prognostic value of p53 protein expression and clinicopathological factors in infiltrating ductal carcinoma of the breast. A study of 192 patients. Histol Histopathol 16: $99-106$

Teel P, Sommers SC (1964) Vascular invasion as a prognostic factor in breast carcinoma. Surg Gynecol Obstet 118: 1006-1008

Thor AD, Moore II DH, Edgerton SM, Kawasaki ES, Reihsaus E, Lynch HT, Marcus JN, Schwartz L, Chen LC, Mayall BH, Smith HS (1992) Accumulation of p53 tumour suppressor gene protein: an independent marker of prognosis in breast cancers. J Natl Cancer Inst 84: $1845-1855$

van de Velde CJH, Gallager HS, Giacco GG (1986) Prognosis in nodenegative breast cancer. Breast Cancer Res Treat 8: 189-196

Van Hoef MEHM, Knox WF, Dhesi SS, Howell A, Schor AM (1993) Assessment of tumour vascularity as a prognostic factor in lymph node-negative invasive breast cancer. Eur J Cancer 29: $1141-1145$

Weidner N, Semple JP, Welch WR, Folkman J (1991) Tumour angiogenesis and metastasis-correlation in invasive breast carcinoma. $N$ Engl J Med 324: $1-8$

Weigand RA, Isenberg WM, Russo J, Brennan MJ, Rich MA (1982) Blood vessel invasion and axillary lymph-node involvement as prognostic indicators for human breast cancer. Cancer 50: $962-969$

Wright C, Angus B, Nicholson S, Sainsbury JRC, Cairns J, Gullick WJ, Kelly P, Harris AL, Horne CHW (1989) Expression c-erbB-2 oncoprotein: a prognostic indicator in human breast cancer. Cancer Res 49: 2087-2090

Wyllie AH, Beattie GJ, Hargreaves AD (1981) Chromatin changes in apoptosis. Histochem J 13: 681-692

Zhang GJ, Kimijima I, Abe R, Watanabe T, Kanno M, Hara K, Tsuchiya A (1998) Apoptotic index correlates to bcl-2 and p53 protein expression, histological grade and prognosis in invasive breast cancers. Anticancer Res 18: $1989-1998$ 